\title{
Attitudes Towards Disabled Children:- A Qualitative Study In Rural Region of Maharashtra.
}

\author{
Shende Mahendra L ${ }^{1}$, Phalke Vaishali $D^{2}$ \\ ${ }^{I}$ Associate Professor,Dr APJAK, College Of Physiotherapy \\ ${ }^{2}$ Professor, Dept Of PSM, Rural Medical College, PIMS.
}

\section{Introduction}

Over the last 60 years, India has evolved into an important player in the international arena. During this time, it has achieved a number of accomplishments including becoming a leader in computer and communications technology. Even more impressive, India boasts one of the fastest growing economies in Asia.

Despite such progress, $25 \%$ of the population lives below the poverty line, $40 \%$ of the population is illiterate and approximately $70 \%$ of the population live in rural areas and lack access to adequate medical care and support services. Furthermore, India is still very enveloped by cultural standards and norms that have prevailed for hundreds of years. In a society of competing dualisms between rich and poor, urban and rural, developed and undeveloped, it is not hard to imagine the conditions and challenges that the most vulnerable members of society face.The predominant religion in India is Hinduism, practiced by more than $80 \%$ of the population. Hindu's believe that an individual will be reborn or re-incarnated after death until they have attained salvation. Children are the perfect extension and expression of a couple's love and caring (Gibran, 1986). The emotional preparation for expectant parents is usually shaped by a glamorous image of the baby, a kind of ego ideal. The discrepancy between the perfect child of their fantasy and the real child may be the cause for negative attitudes and parenting stress. Often a range of emotions, such as denial, guilt, blame, frustration, anger and despair, sweep through the parents as they are confronted by their children with disability (Bhan, 1995).

Loss of hope for the 'perfect child' causes grief, and over time the feeling is heightened by loneliness, isolation, and exhaustion. Parents frequently over-protect their child and feel guilty that they are responsible for the child's disability.Various studies have focused on stressors associated with caring for children with disabilities, and the deleterious effects on parents' well-being.

There is evidence that family attitude contributes to prognosis in these children. Limited financial resources, lack of appropriate services, and insufficient support systems are the family system risk factors that can contribute to poor prognosis (Singer \& Powers, 1993). There have been efforts internationally to include children with disabilities in the educational mainstream. Geoff Lindsay (2007:1) suggests that inclusive education/mainstreaming is the key policy objective for education of children and young people with disabilities'.Inclusive education entails 'increasing the participation of students in, and reducing their exclusion from, the cultures, curricula and communities of local schools' (Booth and Ainscow, 1998:2). The Salamanca Statement and Framework for Action on Special Needs Education (1994) adopted by the World Conference on Special Needs Education paved the way for inclusive education. It upheld the aim of 'education for all' by suggesting some foundational changes in programmes and policies of nations. The Statement solicits governments to give the highest priority to making education systems inclusive and adopt the principle of inclusive education as a matter of law or policy.It emphasizes that every child has a basic right to education and every child has unique characteristics, interests, abilities and learning needs.

The Salamanca Statement maintains that 'inclusion and participation are essential to human dignity and the enjoyment and exercise of human rights' (quoted in CSIE 1997). Thus we see inclusive education as largely emanating from the human rights perspective which upholds that variations in human characteristics associated with disability, whether in cognitive, sensory, or motor ability, as inherent to the human condition and such conditions do not limit human potential (Rioux and Carbet 2003). The idea of children having rights independently of the adults around them is a relatively new concept of the past century and a common theme in early legislation was that children were seen as passive recipients, to be 'seen but not heard' (Munro 2001). The adoption of Convention on the Rights of the Children (CRC) in 1989 and the World Summit for Children in 1990 were promising enactments and it appeared that rights of children were seriously being considered by the governments and international community (International Save the Children Alliance 2001).

The rights of the children were envisaged at the CRC and reaffirmed through the recent UN Convention on the Rights of Persons with Disabilities (UNCRPD) (2006). The CRC remains a landmark document which comprehensively covers civil and political, social, economic and cultural rights of children.It takes due consideration of the survival, development, protection and participation needs of children. India ratified the convention in December 1992, thereby committing itself to protect and promote rights of all its 
children. Both the conventions clearly uphold the importance of education of all children with disabilities and maintain that they must not be excluded from the general educational system. Though the member nations have ratified the convention there are reported good practices and violations all over the world. 'Disability' in children renders them even more vulnerable to violation of their rights. Children with disabilities have universally suffered discrimination, violence and abuse, poverty, exclusion and institutionalization (International Save the Children Alliance 2001:2). Jones' (2000) critic of the 'country reports' to the UN Committee on the Rights of the Child, is that in majority of the cases children with disabilities were referred under Article 23 only focussing on rehabilitation and special care.

Children were rarely mentioned under Article 28 (the right to education) and other Articles, indicative of 'welfare' rather than 'rights' approach of nations towards children with disabilities. While existing literature has focused on family impact and stressors involved in taking care of children with disabilities, the current qualitative study explore how parents, teachers and classroom assistants in Rural region of Maharashtra view children with communication impairments, the reasons they give for their difficulties, and how they currently help them. This included an exploration of any Alternative and Augmentative Communication (AAC) methods used. AAC allows people to communicate without using speech, for example by signing or using pictures. The overall aim was to generate solutions and recommendations to remove any barriers preventing these children from communicating effectively and potentially to improve their quality of life.Data was collected through 18 semi-structured interviews (with parents, classroom assistants and teachers), and one focus group discussion with teachers, across rural region of Maharashtra.

\section{Models And Beliefs About Disability In India}

Assistive technology is commonplace in high income countries, and refers to any rehabilitative devices for people with disabilities; e.g. wheelchairs, braille and hearing aids. Aids for people who have little or no speech are called Augmentative and Alternative Communication (AAC) and therefore are forms of assistive technology (see glossary). These range from "low tech" methods such as communication boards with pictures to 'high tech' systems including computer aided devices which produce an "electronic voice".

Before introducing any form of assistive technology into a culture, it is important to study the explanation given to the cause of disability, within that cultural context (Ripat et al 2010). Local explanations for communication impairments could be at an individual level based on the biomedical model, at a social world level where disability is the result of somebody wronging another person in the community, or there could be a supernatural explanation involving witchcraft (Ripat et al 2010).

The explanation of the causes of impairment determines how disabling it is in that context. This links to the social model of disability which distinguishes between impairment, which is the actual "deficit" or difficulty that the person has, and the disability, which is how society responds to people with impairments (Shakespeare 2012). In its purest form, this model states that people with disabilities face difficulties because of discrimination and lack of services .Society should change and remove these barriers, rather than the people with impairments, who should just be seen as being part of natural human diversity.However, in India, rehabilitation and the use of AAC may only be adopted if it fits in with the families' explanation of the impairment. So whilst it fits well if the explanation is biomedical, meaning that the child requires help to become independent, if the impairment is seen as shameful, then it may be more difficult to introduce rehabilitation aids aiming to give the child a voice (Ripat et al 2010). There is only limited research looking at communication disabilities and the role of traditional healers and traditional beliefs in India. Cultural beliefs are often mistakenly believed to be the cause of a disabled child's neglect or abuse, when actually socio-economic factors may be the root cause. It is often a question of priorities: in a situation of hunger, disease and extreme poverty, the disabled child is often weaker and less of a priority to send to school, and it can be difficult to take them to appointments with a large family to look after (Ingstad 1990).

\section{Multicultural Issues in AAC research}

As the study of AAC has grown as a discipline, recognition of the need to gather information from multi-cultural settings has increased, but challenges remain. Matsumo (1994) describes the three main challenges: allowing our own culture to affect how we see the others' behaviour, creating representations of how we think others should behave rather than how they actually behave, and creating cultural stereotypes. Reflexivity is therefore crucial in this type of research ( Bridges 2004).

The overall culture of the family is particularly important. Hetzroni et al (1996) created guidelines for cultural assessment intervention, to find out what aspects of the language and culture lend themselves to AAC. Parette et al (2004) highlighted further cultural considerations when working with children with communication disabilities and their use of AAC. It is imperative that the child's AAC device is matched to their natural environment, in terms of everyday activities. Furthermore, it may be preferable for a child to learn a sense of duty and collective support, rather than learning to be independent (Parette et al 2004). A final point regarding 
multi-cultural issues is that it is important to adapt training programmes for local populations, to suit the cultural context ( Kent Walsh 2003).

\section{Disability issues and AAC use in Rural Region of Maharashtra.}

A literature review was conducted to investigate disability issues within rural region of Maharashtra of India, particularly in relation to communication disabilities and AAC. In a survey of rehabilitation services and how people cope with disability in rural region of Maharashtra were interviewed and found access to assistive devices was a major indicator of the participants' quality of life, and the main barrier to this was financial. Those without assistive devices for mobility had to beg, whereas those with assistive devices were able to gain access to education and employment.

They looked at the traditional views and issues associated with child disability in rural region of Maharashtra. Challenges faced by families included the time needed to look after the child, the lack of funds for food, treatment and transport costs, parental stress and resulting health problems. Communication problems emerged as a major challenge, with several carers complaining that their children showed challenging behaviour because they had no way to communicate. Many respondents mentioned that negative attitudes towards children with disabilities were rife in their communities. Observational data confirmed this, as many children in the study were observed hidden in back rooms. As with other parts of India, parents often believed that the cause of their child's disability was God's will, or due to spirits and witchcraft. Most families in the study accepted their children in the family setting and attempted to seek cures both from modern doctors and traditional healers, showing their desire to try to help their children. The overall conclusion from this study was the need for a holistic approach and full collaboration between all stakeholders.

In a survey of AAC use in rural region of Maharashtra found that extreme poverty meant that AAC tended to be given a lower priority on the health and family agenda. Also, families would often assume that rehabilitation was irrelevant as their child's cure was in the hands of fate. Alternatively, they would expect an immediate cure after intervention. Alant (1996) looked at AAC use in developing countries and how to implement effective change, highlighting the fact that people with communication disabilities are often one of the most neglected amongst disabled groups. Alant (1999) also conducted a prevalence study of students with little or no functional speech in special needs settings in Pretoria, South Africa. Only $1 \%$ of the children in this category had been exposed to AAC methods.

More recently, Mukhopadhyay et al (2009) looked at AAC use in Botswana, focusing on teachers' perceptions regarding AAC use in their classes and their knowledge, skills and attitude towards using AAC for their non-speaking learners. Child related challenges included the difficulty of understanding children with limited speech, knowing how to discipline them, and also knowing what developmental level they were functioning at. Additional challenges included the stigma of working in a special needs school, the amount of work involved, and the lack of time and resources available for implementation (Mukhopadhyay et al 2009). The recommendations were for more support staff in special needs classrooms, more culturally appropriate resources and more of a focus on teamwork within schools through training (Mukhopadhyay et al 2009).

\section{Global Findings - Barriers and Facilitators to AAC use}

The majority of global findings regarding AAC originate from research in high income countries, although only those including low tech AAC are discussed below. In a review, Baxter et al (2012) found that barriers included the parents not being involved enough during the decision making process (McNaughton et al 2008) and negative family attitudes towards the AAC system (Johnson 2006). The implications are that attitudes and input of the people surrounding the child using AAC are crucial to its successful implementation.

The issue of insufficient time was highlighted as a barrier throughout the literature: to make and create resources, for training, and for the child. Indeed lack of time and lack of flexibility from teachers and other professionals can prevent AAC from working at all (Feiler et al 2010). There also needs to be an individual approach to developing AAC for each child, requiring a close relationship with teaching assistants, under supervision from professionals. The final barrier to highlight is insufficient resources to fund teaching assistants, and insufficient training to deliver an individual approach to AAC. Therefore, a key factor to help disabled children use AAC more effectively is training for staff and parents. Schlosser et al (2000) evaluated a training package for staff working with a child using an AAC system, and described positive outcomes from the training. The need for more training for both parents and professionals was also found in studies by Lund and Light (2007) and Goldbart and Marshall (2004).

Teachers also need to be multi-talented with sufficient creativity, patience, flexibility, and practical AAC knowledge and skills (Soto et al 2001). Without this, it is unlikely that their students who need AAC will develop appropriately. Teachers' attitudes were noted to be particularly important. Often when teachers realise the value of AAC for improving the students' behaviour in addition to their communication skills, this improves their attitude towards its use. Theoretical training should therefore be combined with attitudinal change. De 
Bortoli et al (2010) cite a number of studies where teachers showed no significant improvement in their observed interaction skills after training but a more important positive correlation was between teachers' attitudes and the amount of interaction they had with their students. A recommendation in the study was for hands on training to take place with the students with communication disabilities based on interactional skills, rather than giving theory based training alone.

Attitudes of parents, teachers or assistants are often part of a bigger picture, and not just their individual viewpoints. De Bortoli et al (2010) discussed "complexity theory" meaning that systems are built in layers, with different contextual factors taking place on each level: the school level is in turn embedded at district level, and then at institutional and national levels. These levels are not isolated, but are inter-connected in many complex ways. This is similar to Bronfemmer's (1997) ecological theory, with five embedded systems.

\section{Summary}

This review has discussed the real need for qualitative research about children with communication disabilities in rural region of Maharashtra, and why this is a timely piece of research. To set the scene and the context for this research, traditional beliefs regarding disability in India were summarized. Alternative and Augmentative Communication (AAC) has been discussed, particularly by looking at multicultural issues regarding its use. Whilst there is a plethora of studies looking at the facilitators and barriers towards the use of AAC in developed countries, there is a real dearth of such literature in India. Studies have focused on how Indian families cope with disabled children, and their attitudes and beliefs about disability. This study would add to this body of knowledge by contributing a specific focus on communication disabilities and attitudes towards using different methods of communication.It is important to look at any current cultural, practical and attitudinal barriers preventing children from accessing AAC to make the best use of this new technology.

\section{Research Questions}

1. What are the attitudes, beliefs and explanations of parents and professionals regarding communication disability in children, and how do they feel these children are viewed by society?

2. How do the participants currently help the children with communication disabilities to communicate? What are the perceived barriers to or facilitators helping them to do this effectively?

The overall aim is to generate solutions and recommendations for the removal of any barriers to helping these children from communicating effectively and potentially improve their quality of life.

\section{Methodology}

This study consisted of primary research in rural region of Maharashtra.

\section{Design}

A qualitative interview methodology was chosen as there has been no previous research looking at school staff and parents' attitudes and beliefs regarding children using AAC, so this was a preliminary investigation to explore the issues. This methodology gave the participants opportunity to provide rich descriptions of their own specific contexts, which gave a more in-depth level of analysis. It also adds to previous qualitative research looking at beliefs of parents regarding causation of disability, and attitudes towards disability (Hartley 1995).

\section{Target and Study Population}

The target population for this study was children with communication disability, and the study population were parents, teachers and classroom assistants of such children. The recruitment of the participants in this study was achieved initially by direct e-mail contact with the directors of centers in rural region of Maharashtra and subsequent face to face meetings. A purposive sampling method was used to select 6 teachers, assistants and parents, with guidance from the directors of the centers who ensured that there was as much diversity in the socio-economic and educational backgrounds of the participants as possible. Purposive sampling improves the chance of research generating meaningful results (Green et al 2009).

The main inclusion criteria for teachers and assistants, was that they had daily contact with at least one child with a severe communication disability. All participants were given an information sheet in English. The use of a translator was offered but not necessary, as all the participants chose to converse in English. It was emphasized that they would be free to leave the study at any time and were under no obligation to take part, and that it would not affect any services they receive if they did not wish to take part. The participants were also asked to sign a consent form acknowledging their consent to take part in the study. A separate written consent form was also gained from all the participants consenting to the audio-recordings. 


\section{Conclusion}

Being able to communicate is a fundamental human right. It is only through communication that a person's basic needs can be met. In addition to the very real link with improving educational performance and economic prospects (Coleridge 2007), improving communication skills can also be linked to child protection through improving a child's ability to report abuse ( Ellery et al 2011). Therefore it is crucial to understand the context in which children with communication disabilities live in low income countries.

Research Question 1: What are the attitudes, beliefs and explanations of parents and professionals regarding communication disability in children, and how do they feel these children are viewed by society?

The data showed that traditional beliefs are still common in the urban/ rural settings of Maharashtra, replicating findings in rural settings seen in Hartley's (2005) study. Explanations for a child's communication disability included witchcraft, taboos, Gods, incorrect diet, woman's fault, use of family planning pills and upbringing of the child.

Western or biomedical views were also prevalent in the data, but these views were less common .The data also showed that most parents and professionals feel that these children are viewed negatively in society (appendix v). Traditional views did not necessarily give rise to negative consequences, as it sometimes led to positivity. However the embarrassment of having a child with a communication disability may still lead parents to hide that child away even if they understand the cause as being bio-medical. The most common finding was that families tried a combination of treatments, often starting with traditional cures and then later adopting western treatments (appendix v). Therefore, if traditional healers were able to refer families onto therapy and schools as early as possible when families first come to them, and give basic advice to parents, it may help that child to reach his/ her communicative potential. It is well documented that early intervention is best (Ljubesic 2012).

Research Question 2: How do the participants currently help the children with communication disabilities to communicate? What are the perceived barriers or facilitators to help them to do this effectively?

The formal AAC methods used were objects of reference, picture pointing, picture exchange, communication books, sign language, and writing ( see glossary). Other non-formal methods included handleading, eye pointing, behaviours, noises, and whole body movements. Formal methods have more potential for helping the children to develop their functional communication skills. Teachers described using formal methods double the number of times that parents or assistants did (appendix v). Parents themselves acknowledged their own need to understand more about what to do, describing a lack of knowledge about how to help as a key problem. All three respondent groups agreed that lack of time, and the child's own limitations were barriers to helping, and teachers and parents in particular agreed that lack of money and resources was a key problem.

The "Changing Attitudes" section highlights some of the facilitators to help children with communication disabilities to use AAC more effectively (see model 1 and table 3 ). The findings have highlighted that it is important not to separate tackling disability awareness-raising in society, from more specific training programmes to help these children to use low tech AAC more effectively. By using "complexity" and "ecological theory" we have seen how national views are embedded across society. It is important to run campaigns at a national rather than a local level, and to involve national ministries in the implementation of the policies. Also, by looking at how parental coping strategies can impact on a child's outcome, and potentially their ability to communicate, the findings suggest that there should be a larger focus on mental health services, counselling and parent support groups. Finally, by highlighting the issue of abuse in these children and their inability to report such crimes this becomes a child protection issue. This is another way the communication disability issue can make it onto the mainstream agenda, rather than being side-lined as an issue of little importance.

\section{Recommendations for Action in the light of the data}

- For Local District Councillors ( LDCs) to support parents training seminars and sessions, and the adoption of parent groups for children with communication disabilities.

- For Local Education Councils to implement inclusive education in their districts, and increase training on communication disabilities for both special needs and mainstream teachers.

- For the National Government to back a nation-wide media campaign to raise awareness of communication disabilities and the impact on society.

\section{Limitations}

Data saturation was almost reached by the last interview, so doing additional interviews may not have yielded much new information. However, other benefits would have been to further substantiate previous data, 
and to allow further focus group discussions to have taken place in order to help triangulate the data. With more time, it would have been interesting to include more detailed observations of what happens within the home or school environment, for additional triangulation. It has also only been possible to analyse the main themes to emerge, rather than the differences between the individual respondents' attitudes and views, and their demographics.

\section{References}

[1]. Afako R, Hartley S, Ojwang P and Wairimu C (2001) Implementation of inclusion education policies in Uganda Centre of International Child Health and Uganda National Institute of Special Education Publication.

[2]. Alant E (1996) Augmentative and Alternative Communication in Developing Countries : Challenge of the Future Journal for Augmentative and Alternative Communication $12: 1-12$

[3]. Alant E (1999) Students with little or no functional speech in schools for students with severe mental retardation in South Africa Journal of Augmentative and Alternative Communication 15: 83 - 92

[4]. Andrade V and Ross E (2005) Beliefs and practices of Black South African traditional healers regarding hearing impairment International Journal of Audiology (44): 489- 499

[5]. Baxter S, Enderby P, Evans P and Judge S ( 2012) Barriers and facilitators to the use of high-technology augmentative and alternative communication devices : a systematic review and qualitative synthesis International Journal of Language and Communication Disorders 47 (2): 115-119

[6]. Bondy A and Frost L (2001) A Picture's Worth : PECS and Other Visual Communication Strategies in Autism, Woodbine House Publishers, UK.

[7]. Bridges S (2004) Multicultural Issues in Augmentative and Alternative Communication and Language: Research to Practice Journal of Language Disorders 24 (1): 62 - 75

[8]. Bronfenbrenner U (1997) Toward an experimental ecology of human development American Psychologist 32: 513-531

[9]. Calculator S and Black T (2010) Parents' Priorities for AAC and Related Instruction for their Children with Angelman Syndrome Journal of Augmentative and Alternative Communication 26 (1): 30-40 